\title{
Development of a GPU-accelerated MIKE 21 Solver for Water Wave Dynamics
}

Aackermann, Peter Edward ; Pedersen, Peter Juhler Dinesen; Engsig-Karup, Allan Peter; Clausen, Thomas; Grooss, Jesper

Published in:

Facing the Multicore-Challenge III

Link to article, DOI:

10.1007/978-3-642-35893-7_15

Publication date:

2013

Link back to DTU Orbit

Citation (APA):

Aackermann, P. E., Pedersen, P. J. D., Engsig-Karup, A. P., Clausen, T., \& Grooss, J. (2013). Development of a GPU-accelerated MIKE 21 Solver for Water Wave Dynamics. In Facing the Multicore-Challenge III: Aspects of New Paradigms and Technologies in Parallel Computing (pp. 129-130). Springer. Lecture Notes in Computer Science Vol. 7686 https://doi.org/10.1007/978-3-642-35893-7_15

\section{General rights}

Copyright and moral rights for the publications made accessible in the public portal are retained by the authors and/or other copyright owners and it is a condition of accessing publications that users recognise and abide by the legal requirements associated with these rights.

- Users may download and print one copy of any publication from the public portal for the purpose of private study or research.

- You may not further distribute the material or use it for any profit-making activity or commercial gain

- You may freely distribute the URL identifying the publication in the public portal 


\section{DEVELOPMENT of a GPU-ACCELERATED MIKE 21 SOLVER for WATER WAVE DYNAMICS}

Peter Edward Aackermann ${ }^{1}$, Peter Juhler Dinesen Pedersen ${ }^{1}{ }^{\star}$, Allan Peter Engsig-Karup ${ }^{1}$, Thomas Clausen ${ }^{2}$, and Jesper Grooss ${ }^{2} \star \star$

1 Technical University of Denmark (DTU), Department of Informatics and Mathematical Modelling, 2800 Kgs. Lyngby, Denmark

2 DHI Group, 2970 Hoersholm, Denmark

\section{Motivation}

With encouragement by the company DHI are the aim of this B.Sc. thesis ${ }^{3}$ to investigate, whether if it is possible to accelerate the simulation speed of DHIs commercial product MIKE $21 \mathrm{HD}$, by formulating a parallel solution scheme and implementing it to be executed on a CUDA-enabled GPU (massive parallel hardware).

MIKE $21 \mathrm{HD}$ is a simulation tool, which simulates water wave dynamics in lakes, bays, coastal areas and seas and it is one of DHIs most applied commercial products. For this reason a drastic improvement in simulation speed has the potential to change the type of optimization problems where MIKE $21 \mathrm{HD}$ is applicable and thereby open new market segments for DHI.

\section{Model Equations and Discretization}

MIKE $21 \mathrm{HD}$ simulates water wave dynamics by solving a set of hyperbolic partial differential equations called shallow water equations which are given as

$$
\begin{gathered}
\frac{\partial \zeta}{d t}+\frac{\partial p}{\partial x}+\frac{\partial q}{\partial y}=\frac{\partial d}{\partial t} \\
\frac{\partial p}{\partial t}+\frac{\partial}{\partial x}\left(\frac{p p}{h}\right)+\frac{\partial}{\partial y}\left(\frac{p q}{h}\right)+g h \frac{\partial \zeta}{\partial x}+\frac{g p \sqrt{p^{2}+q^{2}}}{C^{2} h^{2}}=0 \\
\frac{\partial q}{\partial t}+\frac{\partial}{\partial y}\left(\frac{q^{2}}{h}\right)+\frac{\partial}{\partial x}\left(\frac{p q}{h}\right)+g h \frac{\partial \zeta}{\partial y}+\frac{g q \sqrt{p^{2}+q^{2}}}{C^{2} h^{2}}=0
\end{gathered}
$$

The solution scheme used is the Alternating Direction Implicit (ADI) method, which results in many tri-diagonal matrix systems, which have to be solved efficiently for each time step.

\footnotetext{
* For contact the authors: s093066@student.dtu.dk and s093053@student.dtu.dk

** We want to thank Allan Peter Engsig-Karup, Thomas Clausen and Jesper Grooss for supervision and support throughout the project.

${ }^{3}$ B.Sc. thesis: http://www2.imm.dtu.dk/pubdb/views/publication_details.php?id=6367
} 


\section{Solution Approach}

Two different parallel solution schemes are implemented. The first $(S 1)$ solves each tri-diagonal system in parallel using a single CUDA thread for each system. This approach use the same tri-diagonal solution algorithm as MIKE $21 \mathrm{HD}$, the Thomas algorithm. The other solution schemes $(S 2)$ adds more parallelism into the system by using several threads to solve each system in parallel. In order to do this efficient are several parallel solution algorithms investigated. The focus have been on the Parallel Cyclic Reduction (PCR) algorithm and a hybrid algorithm of Cyclic Reduction (CR) and PCR.

\section{Results}

We discover that $S 2$ are beneficial for small problems, while $S 1$ yields better results for larger systems. We have obtained $42 \mathrm{x}$ and $80 \mathrm{x}$ speedup in doubleprecision for $S 1$ and $S 2$ respectively, compared to a representative sequential C implementation of MIKE 21 HD. For comparison can a $3072 \times 3072$ system be solved in double-precision on the GPU twice as fast as a $512 \times 512$ system on the CPU. Furthermore, the impact of switching to perform calculation in singleprecision have been investigated. This resulted in $145 \mathrm{x}$ and $203 \mathrm{x}$ speedup for $S 1$ and $S 2$, respectively. We furthermore achieve near linear scaling when using method $S 1$ compared to a quadratic scaling on the CPU.

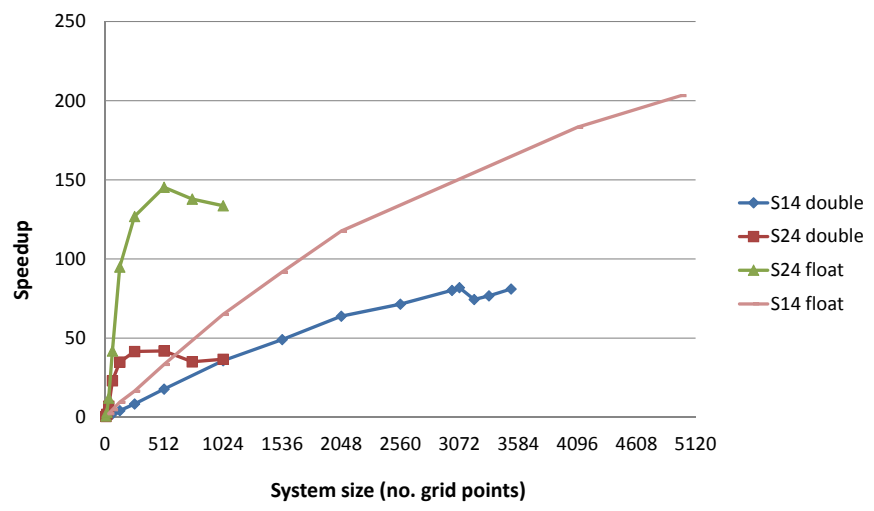

Fig. 1. Speedup of optimized S1 and S2 in single- and double-precision against an implemented CPU version. Result achieved on NVIDIA GeForce GTX 590.

\section{Further research}

An investigating of the precision impact of switching from double- to singleprecision. Especially, using mixed-precision in the core math calculation is anticipated to be beneficial without losing to much precision. 